\title{
Characterization of Metal Coated Diamond Crystallites by Combined Photoacoustic Effect and Photothermal Radiometry
}

\author{
I. Delgadillo-Holtfort ${ }^{a}$, J. Gibkes ${ }^{b}$, M. Chirtoc $^{c}$, \\ E. Neubauer ${ }^{d}$, B.K. Bein ${ }^{b}$ And J. PelzL ${ }^{b}$ \\ ${ }^{a}$ Instituto de Física de la Universidad de Guanajuato, IFUG \\ Apdo. E-143 León, Gto., Mexico \\ ${ }^{b}$ Institut für Experimentalphysik, Ruhr-Universität, D-44801 Bochum, Germany \\ ${ }^{c}$ LTP, UTAP, Université de Reims, BP 1039, 51687 Reims Cedex 2, France \\ ${ }^{d}$ ARC Seibersdorf Research, Dept. Materials Research \\ A-2444 Seibersdorf, Austria
}

\begin{abstract}
Photoacoustic effect and photothermal radiometry were applied to characterise the thermal properties of diamond crystallites which were coated by a $\mathrm{Cu}$ film, a $\mathrm{Cr}$ film and a $\mathrm{Cr}-\mathrm{Cu}$ film. The $\mathrm{Cu}$-coated diamond exhibits a considerable thermal barrier at the $\mathrm{Cu}$-diamond interface which had disappeared for the crystallites with a thin bond $\mathrm{Cr}$ layer between diamond and $\mathrm{Cu}$. The comparison of the responses of both measurement techniques reveals the non-negligible influence of the optical absorption in the visible and IR on the deduced thermal parameters.
\end{abstract}

PACS numbers: 82.47.Pm, 43.35.Ud, 78.20.Nv, 65.40.-b, 66.70.+f

\section{Introduction}

Diamond is the most rigid material with the highest thermal conductivity but it is an electrical insulator. By bringing together a metal and diamond a material with both a high thermal and a high electrical conduction would be achievable [1]. However, when using metal coatings, a thermal contact resistance at the interface generally occurs. One way to reduce this thermal contact resistance is to modify the interface, e.g. by using appropriate coatings. We have set up a research project to investigate the influence of $\mathrm{Cu}$ coatings on the thermal transport properties and optical properties of single coated diamond particles and to study the 
effect of $\mathrm{Cr}$ bond layers between the diamond particle and the $\mathrm{Cu}$ coating. The samples were investigated simultaneously by photoacoustic (PA) effect and photothermal infrared radiometry (PTR). Apart from the different dynamic ranges the comparison of the frequency dependent signals obtained with both methods offer a means to explore the optical properties in the infrared region. The PTR and PA data were analysed in the frame of layer models with and without optical transparency. The frequency behaviours of the PTR signals are compared with those of the PA data and the differences are discussed with regard to the different signal generation processes and optical transparency.

\section{Experimental}

The diamond crystallites with a diameter of about 5-10 $\mathrm{mm}$ were prepared by chemical vapour deposition (CVD) techniques. Some of the crystallites were coated by a $\mathrm{Cu}$ film, a $\mathrm{Cr}$ film, or a $\mathrm{Cu}-\mathrm{Cr}$ double layer. The $\mathrm{Cu}$ films with a thickness of about $30 \mu \mathrm{m}$ were deposited electrolytically. As a consequence of the deposition procedure all $\mathrm{Cu}$ coatings exhibit a roughness on the $\mu \mathrm{m}$ scale. The deposition of the Cr films on the diamond crystallites was performed with PVD/CVD technique. The Cr films, which had a thickness of about $200 \mathrm{~nm}$, were comparatively smooth with a roughness below $100 \mathrm{~nm}$. The double coated crystallites consisted of a Cr film with a thickness of about $200 \mathrm{~nm}$ deposited on the diamond crystallites which then were coated electrolytically with a $\mathrm{Cu}$ film of about $30 \mu \mathrm{m}$ thickness. The aim of the interfacial Cr layer was to improve both the mechanical and the thermal contact between the $\mathrm{Cu}$ coating and the diamond crystallite.

PA and PTR measurements were performed with the same setup based on modulated laser heating in the frequency interval between $100 \mathrm{~Hz}$ and $100 \mathrm{kHz}$ for PTR and $100 \mathrm{~Hz}$ and $2.6 \mathrm{kHz}$ for PA [2]. The crystallites were mounted on a thin glass plate in the middle of the cell without having contact to each other. The signals were normalised with the signals of homogeneous glassy carbon (Sigradur G). Preliminary results of the frequency dependences of the PA phases and amplitudes of the coated and uncoated diamonds had been published elsewhere [3].

\section{Results and discussion}

\subsection{Normalized PA and PTR signal phases and amplitudes}

The frequency dependence of the PTR and PA signals were recorded at room temperature. The raw data were calibrated with the signals of classy carbon (Sigradur) and alternatively with the signals of uncoated diamond on the glass substrate. Here we discuss only the Sigradur normalised data. Sigradur is a black surface absorber that yields the same thermal response for both techniques apart from a constant phase shift. The PTR raw signal amplitude of Sigradur varied roughly with 1 /square $(f)$ whereas that of the PA raw signal decreased with $1 / f$ as expected for a semi-infinite black absorber. The amplitudes and phases of all 
PA signals indicated a roll-off above about $1.5 \mathrm{kHz}$ which is due to the influence of the acoustic resonance of the PA cell at about $2.6 \mathrm{kHz}$. In the whole analysed frequency range of the PA signals of the samples between $100 \mathrm{~Hz}$ and $1.5 \mathrm{kHz}$ the noise level was two orders of magnitude smaller than the signal amplitude. The small amplitude of the glass support in this frequency range prevented from a noticeable contribution of the glass support to the whole PA signal. The phase lags of the PTR signals of all samples started to decrease strongly above about $10 \mathrm{kHz}$. This roll-off behaviour is due to the frequency response of the preamplifier of the IR detector. As above $10 \mathrm{kHz}$ the noise level was also only one order less than the signal amplitudes of the diamond samples only the PTR data in the range $100 \mathrm{~Hz}$ to $10 \mathrm{kHz}$ have been analysed.

In the first approach, the PA data have been fitted by assuming surface absorption and a multilayer structure of the sample. Details are discussed in Ref. [1]. Table shows the deduced thermal parameters. Although the optical properties had not been taken into consideration, some important features could be evidenced. The data of the pure diamond crystallites were described by a

TABLE

Adjusted values from the fit to the photoacoustic data (Fig. 1) compared with literature values. See also Ref. [1].

\begin{tabular}{l|c|c|c|c|c}
\hline \hline & $D[\mu \mathrm{m}]$ & \multicolumn{2}{|c|}{$\begin{array}{c}\text { Thermal diffusivity } \\
\alpha\left[10^{-6} \mathrm{~m}^{2} \mathrm{~s}^{-1}\right]\end{array}$} & \multicolumn{2}{|c}{$\begin{array}{c}\text { Effusivity } \\
\text { n }\end{array}$} \\
\cline { 3 - 6 } & & Exp. & Lit. & Exp. & Lit. \\
\cline { 3 - 6 } & 100 & 50 & 120 & 10000 & 37000 \\
copper & 0.200 & 30 & 30 & 20000 & 20000 \\
chrome & 1 & 0.5 & - & 400 & - \\
interfacial layer & 300 & 500 & 544 & 30000 & 38500 \\
diamond & & & & &
\end{tabular}

two layer model with the diamond as upper layer and the glass support as the lower layer. To adjust the data of the $\mathrm{Cu}$-coated crystallites it was necessary to add a layer between $\mathrm{Cu}$ and diamond. The fitted values of the thickness and of the thermal parameters indicate that this additional interfacial layer is due to a considerable thermal contact resistance between $\mathrm{Cu}$ and diamond. To fit the data of the $\mathrm{Cu}-\mathrm{Cr}$-diamond samples the insertion of an interfacial layer was not required pointing towards an improved mechanical contact and a decreased thermal contact resistance between copper and diamond due to the thin $\mathrm{Cr}$ bond layer. Estimates of the thermal diffusivity and of the effusivity of the $\mathrm{Cu}$ layers of both the $\mathrm{Cu}$-diamond and the $\mathrm{Cu}-\mathrm{Cr}$-diamond samples were obtained on the base of the layer thickness deduced from the evaporation rate. The obtained values are lower by 20 to $40 \%$ compared to the literature values due to a reduced quality of the electrolytically deposited material. 


\subsection{Comparison of PA and PTR signals}

The frequency variation of the normalised phases show roughly the same trend for the PA and PTR response only the magnitude of the effects are different (Fig. 1). With respect to the magnitude of the phase changes the uncoated and the Cr coated diamond exhibit larger phase changes in the PTR response than in the $\mathrm{PA}$ response whereas the situation is the other way round for the $\mathrm{Cu}$ and $\mathrm{Cu}-\mathrm{Cr}$ covered diamond. This observation can be attributed to the partial transparency
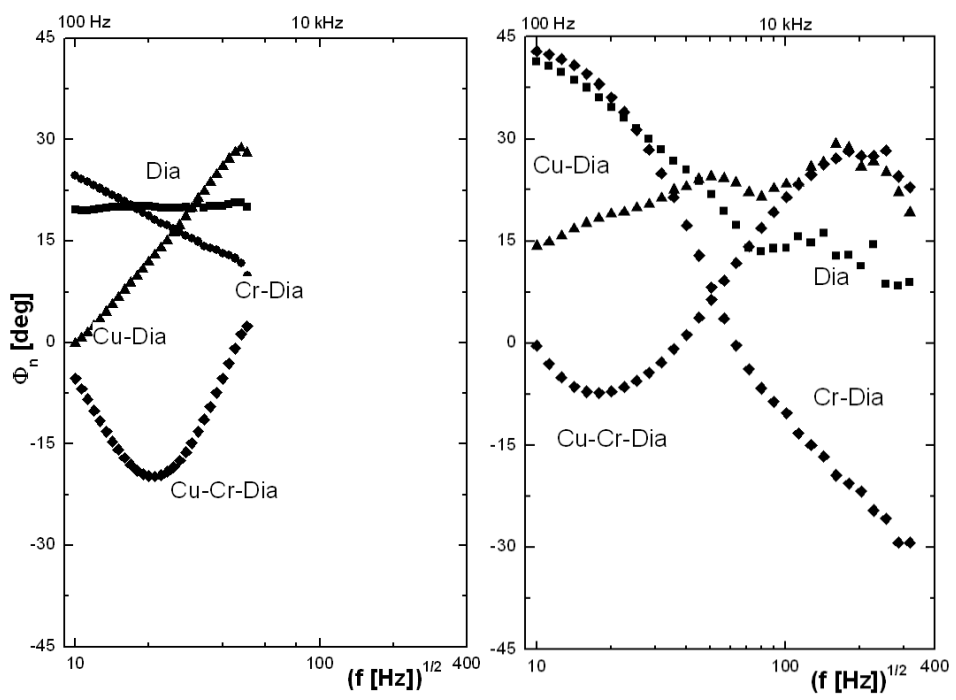

Fig. 1. Normalised signal phases from PA (left) and PTR (right) measurements from non-coated diamond crystallites (squares), Cu-coated diamond (triangles), Cr-coated diamond (circles), $\mathrm{Cu}-\mathrm{Cr}$-coated diamond (rhombs). The reference sample was glassy carbon (Sigradur).

in the optical and the IR region for the non-coated and Cr-coated crystals. As the signal of uncoated diamond is about one order of magnitude larger than that of the glass substrate, the diamond must have a non-negligible absorption in the visible, which is most probably due to small carbon or graphite inclusions as a consequence of a not perfect production process. Simulations of the signals taking into consideration a finite optical absorption for the excitation and for the detection channel show a large sensitivity on both absorption coefficients with respect to the frequency dependence of the signal phase and amplitude. In uncoated and Cr-coated diamond the larger increase in the PTR phase towards lower frequencies can be understood qualitatively by the IR radiation from inside the sample. A quantitative description of the PTR data taking into account visible and IR absorption is in progress. 


\section{Conclusion}

Frequency dependent photothermal measurements have proven to be a suitable tool to explore the bonding of the layers on diamond crystallites. The analysis of the photoacoustic data on the basis of multilayer models already yields first estimates of the thermal parameters and interface contact resistance effects. However, the frequency dependences of the photoacoustic and the photothermal signal phases deviate from each other in distinct frequency ranges varying with the sample which is attributed to the influence of the different IR optical properties on the PTR signal.

\section{References}

[1] Ashok Kumar Dua, in: Materials Science Foundations, Vol. 21, Trans Tech Publications Ltd, Uetikon a.S., Switzerland 2004.

[2] J. Gibkes, M.Sc. Thesis, Faculty of Physics and Astronomy, Ruhr-University Bochum 1991.

[3] I. Delgadillo-Holtfort, E. Neubauer, J.S. Antoniow, J. Gibkes, M. Chirtoc, B.K. Bein, J. Pelzl, Eur. Phys. J. Spec. Top., 153 (2008). 